ABSTRACT ID: 88

Poster(Competing)

\title{
Non-Syndromic Tooth Agenesis: Pattern And Distribution In Permanent Dentition Among IIUM Dental Polyclinic Patients
}

Nurul Hasyiqin Fauzi ${ }^{\mathrm{a}}$ | Widya Lestari ${ }^{\mathrm{b}} \mid$ Azrul Fazwan Kharuddin $^{\mathrm{c}} \mid$ Yunita Dewi Ardini ${ }^{\mathrm{d}}$

${ }^{a}$ Department of Biotechnology, Kulliyyah of Science, IIUM | ${ }^{b}$ Department of Oral Biology, Kulliyyah of Dentistry, IIUM | ${ }^{C}$ Department of Computational and Theoretical Sciences, Kulliyyah of Science, IIUM I Department of Pediatric Dentistry, Kulliyyah of Dentistry, IIUM

Introduction: Non-syndromic tooth agenesis defined as developmental absence of more than one tooth that appears as independent congenital oral trait. Its prevalence, pattern and distribution rates vary by populations. The aim of this study was to identify the pattern and distribution of tooth agenesis in permanent dentition among IIUM dental polyclinic patients. Materials and Methods: 3481 patients aged 12 to 60 years who attended IIUM Dental Polyclinic from April 2016 until April 2017 was evaluated. 33 hypodontia and 2 oligodontia were ruled out. Patients were required to undergo clinical and radiographic examinations. Results: On an average, two teeth were missing per patients. Excluding third molar, the prevalence of population under study was $1.005 \%$. Half of the total cases showed missing a single tooth $(45.7 \%)$, whereas the others ranged from two until nine. The most common missing tooth in maxillary and/or mandibular are lateral incisors $(28 \%)$ followed by second premolars $(26.67 \%)$ and first premolars $(25.33 \%)$. There was no significance difference in missing tooth between male and female $(p=0.48)$. More missing teeth identified at maxilla $(54.67 \%)$ compared to mandible $(45.33 \%)$. More teeth were found missing on the right side $(54.67 \%)$, on the posterior region $(53.33 \%)$ and also unilaterally $(71.42 \%)$. Tooth anomalies such as peg-shaped tooth $(8.57 \%)$ and microdontia $(5.71 \%)$ were found in some cases. Conclusion(s): In conclusion, mild hypodontia is considered as the most common tooth agenesis. The prevalence studied population was less than the previous studies in Malaysia and lateral incisors were identified as the most common missing tooth.

KEYWORDS: tooth agenesis, hypodontia, oligodontia, distribution 